\title{
Microbial Profile and Clinical Outcomes of Fungal Keratitis at a Single-Center Tertiary Care Hospital
}

\author{
Maylander Menard', Yesha S Shah $\mathbb{D}^{2}$, Inna G Stroh ${ }^{2}$, Sidra Zafar ${ }^{2}$, Manjari Sriparna ${ }^{2}$, Nancy Zhang ${ }^{2}$, \\ Ank A Agarwal $\mathbb{D}^{2}$, Nakul Shekhawat ${ }^{2}$, Divya Srikumaran $\mathbb{D}^{2}$, Fasika Woreta $\mathbb{D}^{2}$ \\ 'School of Medicine, Meharry Medical College, Nashville, TN, USA; ${ }^{2}$ Division of Cornea and External Disease, Department of Ophthalmology, The \\ Wilmer Eye Institute, The Johns Hopkins University School of Medicine, Baltimore, MD, USA \\ Correspondence: Fasika Woreta, Division of Cornea and External Disease, Wilmer Eye Institute, Johns Hopkins University School of Medicine, 600 \\ North Wolfe Street, Wilmer B20, Baltimore, MD, 21287, USA, Tel +4I0 955-5650, Email fworetal@jhmi.edu
}

Purpose: To evaluate baseline characteristics, microbiological spectrum, management, and outcomes of patients with culture-proven fungal keratitis.

Methods: Retrospective review of all patients with culture-proven fungal keratitis seen over 6 years at a tertiary referral center.

Results: The present study included 62 eyes from 62 patients. Infection with filamentous organisms was more common than with yeast (66.1\% vs 27.4\%). The most common filamentous organisms were Fusarium (17.7\%) and Aspergillus (16.1\%), while the most common yeast was Candida (24.2\%). The main predisposing factor for filamentous keratitis was contact lens use. Yeast keratitis is most associated with an immunocompromised host and ocular surface disease. Corneal perforation (20.0\%) and surgical interventions (46.8\%) were common, with $27.4 \%$ of eyes requiring at least one penetrating keratoplasty. Filamentous keratitis is more likely than yeast keratitis to require urgent penetrating keratoplasty or enucleation and to receive more than one topical and systemic antifungal agent. Visual outcomes were poor with nearly half of the eyes remaining at 20/200 or worse upon resolution of infection. Worse visual outcomes were associated with poor vision at presentation and a history of ocular surface disease. Antifungal susceptibility testing was not routinely performed, but it demonstrated a relatively high minimum inhibitory concentration for at least one antifungal drug in $90 \%$ of cases when performed (16.1\%) and guided the direction of treatment for $80 \%$ of the cases.

Conclusion: Fungal keratitis is visually devastating. Infections with filamentous fungi predominated over yeast and were generally treated more aggressively both medically and surgically. Filamentous and yeast keratitis had similar durations of infections and visual outcomes. Antifungal susceptibility testing influenced treatment in $80 \%$ of cases in which it was performed.

Keywords: fungal keratitis, mycotic keratitis, filamentous keratitis, corneal culture

\section{Introduction}

Fungal keratitis is a major cause of microbial keratitis and compared to bacterial infections, it is often characterized by a delay in diagnosis, more severe infections, slower response to treatment, and worse visual outcomes. ${ }^{1-5}$ The incidence of fungal keratitis varies strongly based on geography: it is estimated to be responsible for over half of all culture-proven corneal ulcers in tropical countries but remains relatively rare in temperate climates. ${ }^{6,7}$ Similarly, the causative organisms vary geographically with filamentous organisms, such as Fusarium and Aspergillus, predominating in tropical latitudes and yeast being more common in temperate climates. ${ }^{8-10}$ Yeast keratitis is shown to be more common in eyes with preexisting ocular surface disease and reduced host immunity, while filamentous infections are more likely to follow ocular trauma, particularly with organic matter, and to be associated with contact lens wear. ${ }^{8,11,12}$

Clinical suspicion, even in areas where fungal infections are relatively rare, is key to timely diagnosis. However, due to delayed diagnosis and poor response to therapy, medical therapy targeted at the most prevalent organisms in a given location is not always successful and excision of infected tissue may be necessary to control infection. ${ }^{13,14}$ Topical drugs used to treat fungal keratitis include natamycin, voriconazole, and amphotericin B. ${ }^{5}$ Natamycin is considered a first-line treatment for fungal keratitis ${ }^{5}$ with studies showing better outcomes in patients with filamentous fungi compared to 
voriconazole. ${ }^{15,16}$ Although susceptibility testing is not routinely performed and region-specific antifungal susceptibility data are scarce, testing may prove vital in guiding therapy given the recent emergence of drug resistance, especially in cases of severe or recalcitrant infections. Studies of fungal keratitis in the Northeastern and Mid-Atlantic regions of the United States are few and date back only one to two decades. ${ }^{17,18}$ In this retrospective chart review, we aimed to evaluate microbiologic characteristics, management, and outcomes of patients with culture-proven fungal keratitis treated over six years in a single-center, tertiary care academic medical center.

\section{Methods}

\section{Study Population}

All patients seen with microbial keratitis at the Wilmer Eye Institute from August 2012 to June 2018 with a corneal culture positive for fungal organisms were included in this retrospective cohort study. The Johns Hopkins Institutional Review Board approved the study and all work was compliant with the Health Insurance Portability and Accountability Act (HIPAA). Requirement for informed consent was waived due to the retrospective nature of the study. The study met the tenets of the Declaration of Helsinki.

\section{Data Collection}

All corneal cultures performed at the Johns Hopkins Hospital between August 2012 and June 2018 positive for fungi were identified from a microbiology database. A retrospective chart review collected clinical information at presentation and at all follow-up visits for patients with corneal culture positive for fungus and treated with antifungal medications. Collected data included microbiology and pathology data, demographic characteristics, medical and ophthalmic histories, intraocular procedures and surgeries, diagnostic testing, and medications (including dose, frequency, and route of administration) at each clinic visit. Ophthalmologic examinations documented the best-available visual acuity and presence of hypopyon. All data were entered into a computerized data entry form for statistical analysis.

\section{Microbiological Evaluation}

Fungal keratitis was diagnosed based on corneal culture results. Sabouraud dextrose agar, chocolate agar, and blood agar were all used as culture media for corneal cultures. In certain complicated cases with a poor treatment response, the clinician requested sensitivities to guide treatment selection. Drugs that were tested included amphotericin B, voriconazole, natamycin, micafungin, flucytosine, and ketoconazole, although all the drugs were not tested in each case.

\section{Outcome Measures}

Baseline characteristics recorded included potential risk factors for fungal keratitis such as ocular surface disease, prior ocular surgeries, contact lens use, ocular trauma, duration of symptoms prior to presentation at our institution, and therapies received prior to presentation. The best-available visual acuity (VA) was recorded upon presentation and at all follow-up visits. Visual acuity thresholds for low vision (20/50 to 20/160) and legal blindness (20/200 or worse) were used for statistical analysis. Documentation of interventions performed at our institution included topical and systemic antimicrobials, topical corticosteroids, and surgeries such as corneal gluing, corneal biopsy, amniotic membrane graft placement, penetrating keratoplasty, evisceration, and enucleation. Duration of follow-up was defined as time from culture collection to final follow-up. Time to resolution was defined as days elapsed from culture collection to the first visit when the absence of infiltrate was documented on clinical exam. Of note, if the patient did not have a culture performed at the time of presentation, the duration of follow-up and time to resolution were based on the date of culture.

\section{Statistical Analysis}

Differences in proportions of categorical variables were assessed using Chi-square tests and Fisher's exact tests. Differences in continuous variables were compared across groups using the non-parametric Mann-Whitney test. Cox regression models were used to calculate univariate and multivariate odds ratios for VA $<20 / 40$ at the final follow-up visit. A multivariate model analyzing predictors for VA $<20 / 40$ at the final follow-up visit included variables that had a $p$ value of $<0.2$. P values 
of $<0.05$ were considered to be statistically significant. $P$ values were two-sided and nominal. Analyses were performed on STATA 13 (Stata Statistical Software: Release 13; StataCorp LP, College Station, TX, USA).

\section{Results}

\section{Baseline Characteristics}

Over the study period, a total of 70 eyes from 70 patients had a positive corneal culture for a fungal organism, of which, 62 eyes were treated for fungal infection and included in this study. Eight cases were excluded as the fungus was deemed a contaminant based on the clinical judgment of the treating ophthalmologist and because the ulcer responded to treatment with only antibacterial agents. The contamination was documented in the electronic medical record. Patientand eye-specific characteristics of the study population upon presentation are summarized in Table 1 . The median age at presentation was 52.9 years (standard deviation [SD] 21.0). Forty-two percent of patients were women and $66.1 \%$ were Caucasian. At presentation to our institution, the majority of affected eyes $(66.7 \%)$ had VA $<20 / 200$ and therefore met the criteria for legal blindness, $20.0 \%$ had VA $<20 / 50$ and met criteria for low vision, and $13.3 \%$ had vision of 20/40 or better. Twenty-six eyes (41.9\%) had hypopyon, while 5 eyes had dense corneal opacity precluding visualization of the anterior chamber.

On average, patients were symptomatic for 22.8 days (SD 17.1) prior to undergoing corneal culture. Before presenting to our institution, $66.1 \%$ of patients had been evaluated by an ophthalmologist and $32.8 \%$ by a nonophthalmologist. Before presentation $77.4 \%$ of patients had been treated with topical antibacterial medications, $22.6 \%$ were treated with topical corticosteroids either on a chronic basis, and $9.7 \%$ with topical antifungal medications. No patients had been treated with systemic antifungal medications.

\section{Risk Factors for Fungal Keratitis}

Sixty out of 62 patients $(96.8 \%$ ) had at least one ocular risk factor known to be associated with the development of fungal keratitis. Ocular risk factors included a history of recent ocular trauma, history of ocular surface disease, history of ocular surgery, history of eye infections, and contact lens usage. Out of the 2 patients without known ocular risk factors, one had a diagnosis of Type 2 diabetes mellitus and the other was taking systemic immunosuppressive medications. Out of all patients, $58 \%$ were current contact lens wearers, of whom $41.7 \%$ wore contacts for extended periods of time including bandage contact lens; $29.0 \%$ had a history of ocular surface disease; $22.6 \%$ used chronic topical corticosteroids; and $17.7 \%$ had a history of herpetic keratitis. Twenty-five percent of eyes sustained ocular trauma prior to the onset of corneal ulceration, and among these eyes $43.8 \%$ sustained trauma with organic matter. Sixteen percent of eyes had prior penetrating keratoplasty and $30.7 \%$ had a history of other ocular surgeries including cataract extractions, trabeculectomies, and others.

\section{Culture and Sensitivity Results}

Of the 62 eyes with culture-positive fungal keratitis, $66.1 \%$ were infected with filamentous fungi, while the remaining $33.9 \%$ were infected with yeast (Table 2). The most common filamentous organisms were Fusarium (17.7\%) and Aspergillus (16.1\%), while Candida (24.2\%) was the most common yeast. Specific species can be seen in Table 2. More than one microorganism was identified in 26 of the 62 cultures (41.9\%). The most common co-isolated organisms were coagulase-negative staphylococcus (50.0\%) and Propionibacterium acnes (23.1\%). In 22 cases (35.5\%), specimens were submitted to pathology and in 14 of the 22 specimens (63.6\%), results were consistent with a fungal pathogen.

Out of all 62 cases culture-positive fungal keratitis, susceptibility testing was performed in 10 cases (16.1\%), and of these 8 were filamentous fungi and 2 were yeast (Table 2). Relatively high minimum inhibitory concentration (MIC) for at least one antifungal agent was present in 9 of 10 specimens $(90.0 \%)$. MIC data are shown in Supplementary Table A. Results of susceptibility testing guided choice of antifungal therapy in 8 of these 10 cases. The two cases in which susceptibility testing did not affect treatment choice were both infected with Paecilomyces lilacinus, had a relatively milder infection, and had excellent clinical outcomes with final visual acuity of 20/20 and 20/25. In one case, 
Table I Characteristics of Patients and Eyes with Fungal Keratitis at Presentation

\begin{tabular}{|c|c|}
\hline Patient-Specific Characteristics & Total \\
\hline Number of patients (\%) & 62 \\
\hline Gender, \% female (n/N) & $41.9(26 / 62)$ \\
\hline \multicolumn{2}{|l|}{ Race } \\
\hline Caucasian, \% (n/N) & $69.4(43 / 62)$ \\
\hline Black, \% (n/N) & $20.9(13 / 62)$ \\
\hline Median age at presentation, years (SD; range) & $52.9(21.0 ; 0.9-91)$ \\
\hline History of diabetes mellitus, \% (n/N) & $17.7(|1 / 6|)$ \\
\hline Immunocompromised a, \% (n/N) & $8.2(5 / 6 I)$ \\
\hline Duration of symptoms prior to culture, days (SD; range) & $15(17.1 ; 0-69.9)$ \\
\hline History of ocular surface disease ${ }^{b}, \%(n / N)$ & $29.0(18 / 62)$ \\
\hline History of herpetic keratitis, \% ( $\mathrm{n} / \mathrm{N})$ & $17.7(11 / 62)$ \\
\hline Contact lens use ${ }^{c}, \%(n / N)$ & $58.1(36 / 62)$ \\
\hline Extended wear contact lenses, \% (n/N) & $41.7(15 / 36)$ \\
\hline Recent ocular trauma ${ }^{d}, \%(n / N)$ & $25.8(16 / 62)$ \\
\hline Recent ocular trauma with vegetative matter, \% $(\mathrm{n} / \mathrm{N})$ & $43.8(7 / 16)$ \\
\hline Prior use of topical steroids, $\%(\mathrm{n} / \mathrm{N})$ & $46.8(29 / 62)$ \\
\hline Prior penetrating keratoplasty, \% $(\mathrm{n} / \mathrm{N})$ & $16.1(10 / 62)$ \\
\hline History of LASIK, \% (n/N) & $4.8(3 / 55)$ \\
\hline Prior other ocular surgeries ${ }^{e}, \%(n / N)$ & $30.7(19 / 61)$ \\
\hline \multicolumn{2}{|l|}{ Visual acuity at presentation, \% (n/N) } \\
\hline $20 / 40$ or better & $13.3(8 / 60)$ \\
\hline Low vision $(20 / 50$ to $20 / 160)$ & $20.0(12 / 60)$ \\
\hline Legal blindness ( $20 / 200$ or worse) & $66.7(40 / 60)$ \\
\hline \multicolumn{2}{|l|}{ Management prior to/at presentation, $\%(n / N)$} \\
\hline Seen by outside ophthalmologist, \% (n/N) & $66.1(2 I / 4 I)$ \\
\hline Seen by a provider other than ophthalmologist, \% $(n / N)$ & $66.1(2 I / 4 I)$ \\
\hline Topical anti-bacterial, \% (n/N) & $77.4(48 / 61)$ \\
\hline Topical anti-fungal, \% (n/N) & $9.7(6 / 62)$ \\
\hline Systemic anti-fungal, \% (n/N) & $9.7(6 / 62)$ \\
\hline Number of eyes & 62 \\
\hline
\end{tabular}

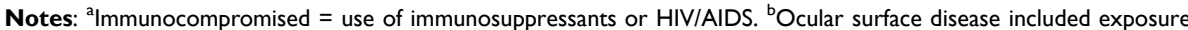
keratopathy, severe dry eye, history of Stevens Johnsons Syndrome, history of ocular pemphigoid, presence of symblepharon, band keratopathy, and epithelial basement membrane dystrophy. ${ }^{\circ}$ Reasons for contact lens use were $75 \%$ refractive, $22 \%$ bandage, $2.8 \%$ cosmetic. ${ }^{\mathrm{d}}$ Recent ocular trauma was trauma temporally associated with current infection. ePrior other ocular surgeries include any ocular surgery (such as cataract extraction, pars plana vitrectomy, glaucoma surgery) other than corneal transplant or LASIK.

Abbreviations: $\mathrm{n}$, number of patients or eyes with characteristic; $\mathrm{N}$, number of patients for whom data are available; LASIK, laser-assisted in situ keratomileusis. 
Table 2 Culture Characteristics

\begin{tabular}{|c|c|c|}
\hline & Percentage & Total $(n=62)$ \\
\hline Filamentous fungi & 66.1 & $4 I / 62$ \\
\hline Fusarium (7 solani, 2 oxysporum, I delphinoides, I species) & 17.7 & $11 / 62$ \\
\hline Aspergillus (4 flavus, 4 fumigatus, I glaucus, I niger) & 16.1 & $10 / 62$ \\
\hline Ramularia species & 9.7 & $6 / 62$ \\
\hline Paecilomyces lilacinus & 8.1 & $5 / 62$ \\
\hline Alternaria species & 3.2 & $2 / 62$ \\
\hline Curvularia (I geniculata, I lunata) & 3.2 & $2 / 62$ \\
\hline Scedosporium species & 3.2 & $2 / 62$ \\
\hline Ascochyta species & 1.6 & $1 / 62$ \\
\hline Beauveria bassiana & 1.6 & $1 / 62$ \\
\hline Epicoccum species & 1.6 & $1 / 62$ \\
\hline Bipolaris species & 1.6 & $1 / 62$ \\
\hline Yeast & 33.8 & $21 / 62$ \\
\hline Candida (7 albicans, 8 parapsilosis) & 24.2 & $15 / 62$ \\
\hline Exophiala species & 4.8 & $3 / 62$ \\
\hline Rhodotorula species & 3.2 & $2 / 62$ \\
\hline Sensitivity testing performed & 16.1 & $10 / 62$ \\
\hline Pathology specimen collected ${ }^{a}$ & 35.5 & $22 / 62$ \\
\hline Fungal pathogen identified on pathology & 63.6 & $14 / 22$ \\
\hline
\end{tabular}

Notes: ${ }^{a}$ All eyes were cultured. These eyes had both a culture and pathology specimen collected. Abbreviations: $n$, number of eyes with characteristic.

susceptibility testing demonstrated the highly resistant fungus Scedosporium prolificans, prompting consultation with the infectious diseases department who recommended enucleation for source control.

Out of the 10 eyes in which susceptibilities were tested, nine (90.0.\%) were treated with more than one topical antifungal medication, seven (70.0\%) received systemic antifungals, and four (40.0\%) received intraocular antifungals. Four $(40 \%)$ of these eyes progressed to perforation; three underwent therapeutic penetrating keratoplasties, while one was enucleated. Visual acuity improved in 7 of 10 eyes; while only one eye had VA $>20 / 40$ or better at presentation, five eyes achieved this level of acuity by the end of follow-up. Out of the three eyes in which acuity did not improve, two were able to perceive light at presentation but lost all perception of light by the end of follow-up (one of these was enucleated), and one eye remained perceptive only to hand motions.

\section{Interventions and Outcomes}

Information on the time to resolution was available for 47 of 62 patients, while the remaining 15 eyes had an active infection at the last follow-up. Duration of follow-up was available for 59 of 62 patients who had at least 1 follow-up visit.

Medical and surgical interventions as well as visual outcomes are shown in Table 3. The mean duration of follow-up at our institution was 14.3 months (SD 17.3). The mean time from culture collection to the resolution of the ulceration, which was available for 47 eyes, was 2.2 months (SD 2.0). Among cured eyes, final VA was $20 / 40$ or better in $40 \%$ ( 24 of 60 ) of eyes and $20 / 200$ or worse in $43.3 \%$ (26 of 60 ) of eyes. 
Table 3 Interventions and Outcomes in the Affected Eyes of Patients with Fungal Keratitis During Follow-Up

\begin{tabular}{|c|c|c|}
\hline Event & Percentage & Total $(n=62)$ \\
\hline \multicolumn{3}{|l|}{ Final visual acuity ${ }^{a}$} \\
\hline $20 / 40$ or better & 40.0 & $24 / 60$ \\
\hline Low vision $(20 / 50$ to $20 / 160)$ & 15.0 & $9 / 60$ \\
\hline Legal blindness (20/200 or worse) & 45.0 & $27 / 60$ \\
\hline Mean duration of follow-up ${ }^{\mathrm{b}}$, months (SD; range) & $14.3(17.3 ; 0-62.3)$ & \\
\hline Mean time to resolution after culture ${ }^{c}$, months (SD; range) & 2.2 ( $2.0 ; 12$ days -8.5 months $)$ & \\
\hline Topical anti-fungal & 96.8 & $60 / 62$ \\
\hline Natamycin & 62.9 & $39 / 62$ \\
\hline Voriconazole & 62.9 & $39 / 62$ \\
\hline Amphotericin & 27.4 & $17 / 62$ \\
\hline Multiple topical anti-fungal agents & 51.6 & $32 / 62$ \\
\hline Voriconazole + Amphotericin & 11.3 & $7 / 62$ \\
\hline Voriconazole + Natamycin & 32.3 & $20 / 62$ \\
\hline Natamycin + Amphotericin & 3.2 & $2 / 62$ \\
\hline All three & 4.8 & $3 / 62$ \\
\hline Systemic anti-fungal ${ }^{d}$ & 27.4 & $17 / 62$ \\
\hline Intraocular anti-fungal & 21.0 & $13 / 6 \mid$ \\
\hline Topical corticosteroid & 33.9 & $21 / 61$ \\
\hline Surgical intervention & 46.8 & $29 / 62$ \\
\hline Corneal transplant & 33.9 & $21 / 62$ \\
\hline Corneal biopsy & 12.9 & $8 / 62$ \\
\hline Amniotic membrane graft & 9.7 & $6 / 62$ \\
\hline Glue to perforated cornea & 6.5 & $4 / 62$ \\
\hline Enucleation or evisceration & 4.8 & $3 / 62$ \\
\hline Multiple surgical interventions & 17.7 & $11 / 62$ \\
\hline
\end{tabular}

Notes: ${ }^{a}$ Final visual acuity indicates visual acuity at last visit. Data available for 60 patients. ${ }^{b}$ Duration of follow-up for keratitis indicates time from date of

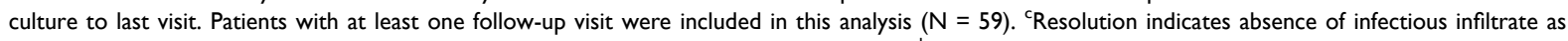
documented in the clinic exam and/or progress note. Information available for $\mathrm{N}=48$ eyes. ${ }^{\mathrm{d}} \mathrm{All}$ systemic anti-fungal medications were of the azole class. Abbreviations: $\mathrm{n}$, number of eyes with characteristic; $\mathrm{N}$, number of eyes for whom data are available.

The vast majority of eyes $(96.8 \%)$ were treated with topical antifungal medications, such as natamycin $(62.9 \%)$, voriconazole $(62.9 \%)$, and amphotericin B (27.4\%). One patient was treated with systemic antifungal medications based solely on the preference of the treating ophthalmologist; one patient was seen only at our institution for the culture collection, thus management information was not available. Nearly half of eyes (46.8\%) were treated with a single topical antifungal agent, while $51.6 \%$ of eyes were treated with more than one antifungal agent. Systemic antifungal medications of the azole class were used in $27.4 \%$ of cases. Intra-ocular antifungal medications including intrastromal, intracameral, and intravitreal formulations were used in $21.0 \%$ of cases, usually in conjunction with surgical intervention. 
Table 4 Risk Factors for Poorer Outcomes in Fungal Keratitis

\begin{tabular}{|c|c|c|c|c|}
\hline \multirow[t]{2}{*}{ Characteristic at Presentation } & \multicolumn{4}{|c|}{ Final VA Worse Than $20 / 40^{\mathrm{a}}$} \\
\hline & Crude OR (Cl) & $P$ value & Adjusted OR $(\mathrm{Cl})^{c}$ & P value \\
\hline Gender (reference female) & $1.57(0.55-4.46)$ & 0.396 & & \\
\hline Race (reference white) & $1.23(0.32-4.76)$ & 0.763 & & \\
\hline Age & $1.04(1.01-1.07)$ & $0.01 *$ & $0.99(0.91-1.07)$ & 0.80 \\
\hline Prior trauma & $0.69(0.2 \mathrm{I}-2.26)$ & 0.544 & & \\
\hline Trauma with vegetative matter & $0.45(0.09-2.24)$ & 0.333 & & \\
\hline History of ocular surface disease & $7.86(1.60-48.61)$ & $0.01 I^{*}$ & & \\
\hline \multicolumn{5}{|l|}{ History of herpetic disease } \\
\hline Contact lens use & $0.33(0.11-1.03)$ & 0.057 & $0.48(0.002-96.28)$ & 0.78 \\
\hline Extended wear $\mathrm{CL}$ use & $2.75(0.67-11.23)$ & 0.159 & $0.98(0.07-14.07)$ & 0.99 \\
\hline Duration of symptoms prior to culture & $0.99(0.99-1.01)$ & 0.758 & & \\
\hline Topical steroid use & 3.92 & $0.017^{*}$ & $5.17(0.34-78.88)$ & 0.24 \\
\hline History of corneal transplant & $7.67(0.90-65.13)$ & 0.062 & & \\
\hline History of LASIK & $0.31(0.03-3.61)$ & 0.348 & & \\
\hline History of other eye surgeries & $10.39(2.11-51.05)$ & $0.004 * *$ & & \\
\hline Evaluation by outside ophthalmologist prior to presentation & $0.59(0.19-1.86)$ & 0.367 & & \\
\hline Type of organisms (reference filaments) & $2.15(0.65-7.11)$ & 0.211 & & \\
\hline \multicolumn{5}{|l|}{ Visual acuity at presentation } \\
\hline \multicolumn{5}{|l|}{ Low vision $(20 / 50$ to $20 / 160)$ vs $20 / 40$ or better } \\
\hline Legal blindness ( $20 / 200$ or worse) vs $20 / 40$ or better & $16(4.18-61.2)$ & $<0.001 * * *$ & $24.52(1.20-500.13)$ & $0.04 *$ \\
\hline
\end{tabular}

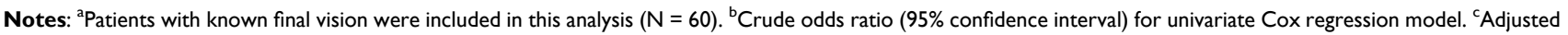
odds ratio ( $95 \%$ confidence interval) for multivariate Cox regression model adjusted for variables with $p$ values $<0.2$ on univariate regression. If a variable with a $p$ value $<0.2$ is not included, the sample size was too small and thus the variable was omitted from the regression. $* p<0.05$. $* * p<0.01$. $* * * p<0.001$.

Abbreviations: VA, visual acuity; OR, odds ratio; $\mathrm{Cl}, 95 \%$ confidence interval; $\mathrm{N}$, number of patients or eyes for which data are available.

Topical steroids were utilized in $33.9 \%$ of cases after the infectious component of the ulceration was deemed controlled as well as for post-operative care.

Twenty-nine (46.8\%) eyes underwent a surgical intervention and eleven (17.7\%) required more than one surgery. Seventeen eyes (27.4\%) underwent at least one penetrating keratoplasty; of these, 13 (20.1\%) eyes underwent urgent therapeutic penetrating keratoplasty for frank or impending corneal perforation, while $4(6.5 \%)$ eyes underwent scheduled transplants for visual rehabilitation. Four of the eyes underwent two keratoplasties during the follow-up interval. Other procedures included corneal biopsy (12.9\%), amniotic membrane grafting $(9.7 \%)$, gluing of the perforated cornea as a temporizing measure $(6.5 \%)$, and eye removal $(4.8 \%)$.

\section{Differences Between Filamentous and Yeast Infections}

Corneal infections with filamentous fungi versus yeast have been shown to be associated with different predisposing risk factors. There were no differences between filamentous and yeast infections in gender $(34.1 \%$ vs $57.0 \%$ female, $\mathrm{p}=$ $0.08)$, age (50.05 vs $57.3, \mathrm{p}=0.52)$, or history of diabetes between the two groups $(19.5$ vs $14.3 \%, \mathrm{p}=0.61)$. Visual 
acuity at presentation between the two groups was similar (LogMAR 1.13 vs $1.12, p=0.95$ ), as was the proportion of eyes with a history of ocular trauma. The filamentous keratitis group had a significantly higher proportion of Caucasian patients compared to the yeast keratitis group $(78.0 \%$ vs $52.3 \%, p=0.038)$. Patients with filamentous keratitis were more likely to have been seen by an outside ophthalmologist before evaluation at our institution $(80.5 \%$ vs $38.1 \%, p<0.001)$ and to have been started on topical antibiotics $(85.4 \%$ vs $61.9 \%, p=0.037)$. Patients with yeast keratitis were significantly more likely than patients with filamentous keratitis to be immunocompromised $(19.0 \%$ vs $0.0 \%, p=$ $0.002)$, take chronic topical corticosteroids $(38.1 \%$ vs $14.6 \%, p=0.037)$, and have a history of penetrating keratoplasty $(33.3 \%$ vs $7.3 \%, p=0.01)$. However, a history of ocular surface disease did not show statistical significance $(19.5 \%$ vs $47.6 \%, p=0.22$ ).

Patients with filamentous keratitis were significantly more likely to wear contact lenses when compared to patients with yeast keratitis $(73.2 \%$ vs $28.5 \%, p<0.001)$. Among the 27 patients who wore contact lenses for refractive purposes, $24(92.6 \%)$ developed filamentous keratitis and two (7.4\%) developed yeast keratitis. Among the eight patients who wore a bandage contact lens in the affected eye, five (62.5\%) developed filamentous keratitis and three (37.5\%) developed yeast keratitis. The one patient who wore cosmetic contact lenses developed filamentous keratitis.

Comparing outcomes in filamentous versus yeast keratitis, a higher proportion of filamentous cases were treated with multiple topical antifungal agents $(60.5 \%$ vs $38.1 \%, p=0.13)$, adjunct systemic antifungal agents $(33.3 \% \mathrm{vs} 11.8 \%, p=$ $0.09)$, and intraocular antifungal agents $(24.4 \%$ vs $11.8 \%, p=0.40)$ but these differences were not statistically significant. Comparing filamentous and yeast keratitis, there were no significant differences in the proportion of eyes developing corneal perforation $(17.1 \%$ vs $23.8 \%, p=0.53)$ or undergoing surgery $(41.5 \%$ vs $57.1 \%, p=0.24)$. Higher proportions of filamentous keratitis cases underwent urgent penetrating keratoplasty for impending or frank corneal perforation (24.4\% vs $33.3 \%, \mathrm{p}=0.45)$ or eye removal $(7.3 \%$ vs $0.0 \%, \mathrm{p}=0.20)$ compared to yeast keratitis. Both groups had a similar mean duration of infection (1.9 vs 2.9 months, $\mathrm{p}=0.07$ ) to resolution after culture. Although the mean final VA was similar across filamentous and yeast keratitis (mean $\log$ MAR $0.70 \mathrm{vs} 0.79, p=0.75$ ), four eyes with filamentous keratitis lost all perception of light and three of these eyes were removed.

\section{Risk Factors for Poor Visual Outcomes}

For the 60 eyes in which final visual acuity was known, a logistic regression model was used to evaluate the association of covariates to VA worse than 20/40 at the last follow-up (Table 4). Covariates with associations having a $p$ value of $<0.2$ on univariate analysis were included in the logistic regression multivariate model. In the multivariate analysis, poor baseline VA (OR $=24.52(1.20-500.13), p=0.04)$ was found to be associated with a final VA worse than 20/40.

\section{Discussion}

This study reviewed nearly 6 years of clinical experience with 62 patients and eyes with culture-proven fungal keratitis to describe presenting features, risk factors, microbiological results, medical and surgical management, and outcomes.

In our cohort, a majority of patients were legally blind with a visual acuity of 20/200 or less. Forty percent of the patients treated for fungal keratitis achieved a final visual acuity of 20/40 or better, while the remainder of patients had a visual acuity of $20 / 50$ or worse. We explored potential causes for these poor visual outcomes, namely a delay in diagnosis, the severity and increased prevalence of filamentous keratitis compared to yeast keratitis, and significant predictors of worse visual outcomes. Additionally, we looked at the usefulness of susceptibility testing.

In our cohort, patients were symptomatic for an average of two weeks before culture collection at our institution, despite the fact that most had seen an ophthalmologist prior to presentation. Furthermore, most patients were treated for presumed bacterial keratitis. The long symptomatic period before presentation to our institution highlights delays in diagnosis of fungal infection, possibly due to low suspicion for the disease. Clinicians may have a lower suspicion of fungal keratitis due to its relative rarity in temperate regions of North America. ${ }^{19}$ However, all of the patients in our cohort had ocular and/or systemic risk factors predisposing them to the development of fungal keratitis. Therefore, in patients with known underlying ocular risk factors, clinicians should have a higher suspicion of fungal keratitis and pursue culture and/or referral to a tertiary care center to expedite diagnosis and treatment. Despite the relative rarity of 
fungal keratitis, improving ophthalmologists' knowledge of the most common fungal isolates within their region and when to suspect fungal keratitis may improve management.

Another potential reason for poor visual outcomes in our cohort is the aggressive nature of filamentous keratitis amongst our patients. Filamentous keratitis is more likely to be treated aggressively compared to yeast keratitis, as evidenced by the use of multiple topical agents and/or systemic and intraocular antifungal medications. Although the final VA was similar between the two groups, a higher proportion of eyes underwent PK, underwent enucleation, or lost light perception in the filamentous keratitis group. One possible reason is the longer duration of infection before culturing (16.4 vs 7.1 days, $p=0.02$ ), indicating delayed diagnosis and possibly more severe infection at the time of referral. Due to the worse visual outcomes amongst patients with filamentous keratitis, it is imperative to treat these patients aggressively and early.

Interestingly, our cohort had a large proportion of filamentous keratitis cases. Although yeast has been shown to be the most common cause of fungal keratitis in temperate regions, ${ }^{8,17,18}$ in our cohort nearly three-quarters of ulcers were caused by filamentous organisms $(66.1 \%$ vs $33.9 \%)$, contrasting from previously published reports from neighboring cities such as Philadelphia ${ }^{18}$ and New York ${ }^{10}$ where yeast keratitis predominated. This discrepancy may be due to changes in the causes of fungal keratitis associated with an increase in contact-lens associated filamentary keratitis, similar to recent observations in London. ${ }^{12}$ While only $29 \%$ and $10 \%$ of patients used contact lenses in the Philadelphia ${ }^{18}$ and New York studies, ${ }^{10}$ over half of the patients in our cohort were contact lens wearers. Furthermore, the declining prevalence of patients with penetrating keratoplasty (PK) due to increased use of endothelial keratoplasty may also be contributing to the decreased incidence of yeast keratitis. Future studies conducted at other centers in the Northeastern and mid-Atlantic United States should assess whether this shift toward increased filamentous keratitis has occurred elsewhere and if contact lens use and PK history are relevant contributors. The larger proportion of filamentous keratitis in our cohort may also partially explain the poor visual outcomes, as it was found to be more aggressive than yeast keratitis.

Predisposing risk factors for filamentous versus yeast keratitis were similar to those previously reported. ${ }^{8,11,12}$ Contact lens wear was more common among patients with filamentous keratitis, while an immunocompromised host, chronic corticosteroid use, ocular surface disease, and a history of corneal transplantation were more common among patients with yeast keratitis. Although studies of fungal keratitis in tropical climates where filamentous keratitis predominates have demonstrated a strong association with ocular trauma, particularly with vegetable matter, our study showed no differences in ocular trauma between filamentous and yeast keratitis. A greater proportion of filamentous keratitis were Caucasian, an association not previously reported. Interestingly, nearly half of the keratitis in our cohort was caused by rare fungal species rather than common ones, such as Candida, Fusarium, and Aspergillus, likely representing the complexity of cases that are referred to a tertiary care center.

Among all patients with fungal keratitis, a VA of 20/200 or worse at presentation was a significant predictor of a final VA worse than 20/40. This is likely because the infection had progressed to a severe state in which treatments were not as efficacious. In patients who present with a low VA, aggressive treatment should be started early due to a higher risk for worse visual outcomes.

The lack of standardized in vitro susceptibility testing that provides a strong in vivo correlation poses another challenge to the medical treatment of fungal keratitis. ${ }^{20}$ Although antifungal susceptibility testing is not routinely performed at our institution, the emergence of drug resistance may render susceptibility testing useful. In our cohort, the 10 cases for which susceptibility testing was performed were more severe (higher rate of hypopyon, perforation, and use of multiple types and routes of antifungal medication), suggesting that the clinicians were inclined to request susceptibility testing for more severe cases. High minimum inhibitory concentration (MIC) for at least one antifungal drug was common and susceptibility testing guided drug choice when performed, but the retrospective nature of our study prevented evaluation of whether any changes in drug choice affected disease course. One study demonstrated that higher MIC for natamycin and voriconazole was associated with a higher risk of corneal perforation but not 3-month visual acuity or scar size. ${ }^{21}$ Although susceptibility testing may not be necessary in every case of fungal keratitis, it may be beneficial in severe or recalcitrant cases as it influences treatment choice. 
This study has limitations due to its retrospective design. Data was recorded as part of routine clinic visits, so there was no standardized recording of risk factors or clinical examination. Since many of the patients were referred, baseline VA was not available, preventing assessment of the effects of fungal keratitis on vision loss. Generalizing results from the tertiary academic medical center setting to other populations must also be done with caution.

\section{Conclusion}

In conclusion, fungal keratitis infections in this study were severe with two-thirds of patients presenting with VA at or below the level of legal blindness, $20 \%$ of eyes developing corneal perforation, and nearly half of the eyes requiring operative interventions ranging from corneal biopsy to eye removal. Infections were prolonged, averaging over 2 months from culture collection to resolution. Filamentous keratitis is more common and severe. Susceptibility testing guided the choice of therapy when performed. Given infection severity and poor visual outcomes, early diagnosis and aggressive treatment of fungal keratitis are paramount.

\section{Acknowledgments}

We would like to acknowledge the faculty and staff of the Wilmer Eye Institute.

\section{Funding}

The Charles Eberhart Research Grant Award 2017, The Oliver Schein Research Grant Award 2018.

\section{Disclosure}

The authors report no conflicts of interest in this work.

\section{References}

1. Wong TY, Ng TP, Fong KS, Tan DT. Risk factors and clinical outcomes between fungal and bacterial keratitis: a Comparative Study. Contact Lens Assoc Ophthalmol. 1997;23(4):275-281.

2. Liesegang TJ, Forster RK. Spectrum of microbial keratitis in South Florida. Am J Ophthalmol. 1980;90(1):38-47. doi:10.1016/s0002-9394(14) 75075-5

3. Miedziak AI, Miller MR, Rapuano CJ, Laibson PR, Cohen EJ. Risk factors in microbial keratitis leading to penetrating keratoplasty. Ophthalmology. 1999;106(6):1166-1171. doi:10.1016/s0161-6420(99)90250-6

4. Prajna NV, Srinivasan M, Lalitha P, et al. Differences in clinical outcomes in keratitis due to fungus and bacteria. JAMA Ophthalmol. 2013;131 (8):1088-1089. doi:10.1001/jamaophthalmol.2013.1612

5. Ansari Z, Miller D, Galor A. Current Thoughts in Fungal Keratitis: diagnosis and Treatment. Curr Fungal Infect Rep. 2013;7(3):209-218. doi:10.1007/s12281-013-0150-1

6. Srinivasan M, Gonzales CA, George C, et al. Epidemiology and aetiological diagnosis of corneal ulceration in Madurai, south India. $\mathrm{Br}$ J Ophthalmol. 1997;81(11):965-971. doi:10.1136/bjo.81.11.965

7. Leck AK, Thomas PA, Hagan M, et al. Aetiology of suppurative corneal ulcers in Ghana and south India, and epidemiology of fungal keratitis. $B r$ J Ophthalmol. 2002;86(11):1211-1215. doi:10.1136/bjo.86.11.1211

8. Shah A, Sachdev A, Coggon D, Hossain P. Geographic variations in microbial keratitis: an analysis of the peer-reviewed literature. $\mathrm{Br}$ J Ophthalmol. 2011;95(6):762-767. doi:10.1136/bjo.2009.169607

9. Galarreta DJ, Tuft SJ, Ramsay A, Dart JKG. Fungal Keratitis in London. Cornea. 2007;26(9):1082-1086. doi:10.1097/ico.0b013e318142bff3

10. Ritterband DC, Seedor JA, Shah MK, Koplin RS, McCormick SA. Fungal Keratitis at the New York Eye and Ear Infirmary. Cornea. 2006;25 (3):264-267. doi:10.1097/01.ico.0000177423.77648.8d

11. Bhartiya P, Daniell M, Constantinou M, Islam FMA, Taylor HR. Fungal keratitis in Melbourne. Clin Experiment Ophthalmol. 2007;70130044246007. doi:10.1111/j.1442-9071.2006.01405.x

12. Ong HS, Fung SSM, Macleod D, Dart JKG, Tuft SJ, Burton MJ. Altered Patterns of Fungal Keratitis at a London Ophthalmic Referral Hospital: an Eight-Year Retrospective Observational Study. Am J Ophthalmol. 2016;168:227-236. doi:10.1016/j.ajo.2016.05.021

13. Keay LJ, Gower EW, Iovieno A, et al. Clinical and microbiological characteristics of fungal keratitis in the United States, 2001-2007: a multicenter study. Ophthalmology. 2011;118(5):920-926. doi:10.1016/j.ophtha.2010.09.011

14. Xie L, Dong X, Shi W. Treatment of fungal keratitis by penetrating keratoplasty. Br J Ophthalmol. 2001;85(9):1070-1074. doi:10.1136/ bjo.85.9.1070

15. Venkatesh prajna N, Krishnan T, Mascarenhas J, et al. The mycotic ulcer treatment trial: a randomized trial comparing natamycin vs voriconazole. JAMA Ophthalmol. 2013;131(4):422-429. doi:10.1001/jamaophthalmol.2013.1497

16. Prajna NV, Mascarenhas J, Krishnan T, et al. Comparison of natamycin and voriconazole for the treatment of fungal keratitis. Arch Ophthalmol. 2010;128(6):672-678. doi:10.1001/archophthalmol.2010.102

17. Xie L, Shi W, Liu Z, Li S. Lamellar Keratoplasty for the Treatment of Fungal Keratitis. Cornea. 2002;21(1):33-37. doi:10.1097/00003226200201000-00008 
18. Tanure MAG, Cohen EJ, Sudesh S, Rapuano CJ, Laibson PR. Spectrum of Fungal Keratitis at Wills Eye Hospital, Philadelphia, Pennsylvania. Cornea. 2000;19(3):307-312. doi:10.1097/00003226-200005000-00010

19. Brown L, Leck AK, Gichangi M, Burton MJ, Denning DW. The global incidence and diagnosis of fungal keratitis. Lancet Infect Dis. 2021;21(3): e49-e57. doi:10.1016/S1473-3099(20)30448-5

20. Sharma S, Das S, Virdi A, et al. Re-appraisal of topical $1 \%$ voriconazole and $5 \%$ natamycin in the treatment of fungal keratitis in a randomised trial. Br J Ophthalmol. 2015;99(9):1190-1195. doi:10.1136/bjophthalmol-2014-306485

21. Lakhani P, Patil A, Majumdar S. Challenges in the Polyene- and Azole-Based Pharmacotherapy of Ocular Fungal Infections. J Ocul Pharmacol Ther. 2019;35(1):6-22. doi:10.1089/jop.2018.0089

Clinical Ophthalmology

Dovepress

\section{Publish your work in this journal}

Clinical Ophthalmology is an international, peer-reviewed journal covering all subspecialties within ophthalmology. Key topics include: Optometry; Visual science; Pharmacology and drug therapy in eye diseases; Basic Sciences; Primary and Secondary eye care; Patient Safety and Quality of Care Improvements. This journal is indexed on PubMed Central and CAS, and is the official journal of The Society of Clinical Ophthalmology (SCO). The manuscript management system is completely online and includes a very quick and fair peer-review system, which is all easy to use. Visit http://www. dovepress.com/testimonials.php to read real quotes from published authors.

Submit your manuscript here: https://www.dovepress.com/clinical-ophthalmology-journal 